\title{
Special Issue on "Intelligent Information Processing: Techniques and Applications"
}

\author{
Lakhmi C. Jain ${ }^{\mathrm{a}, *}$ and Chee Peng Lim ${ }^{\mathrm{b}}$ \\ ${ }^{a}$ University of South Australia, Adelaide, South Australia, Australia \\ ${ }^{\mathrm{b}}$ University of Science, Malaysia
}

In today's digital era, information can be easily acquired from various sources, and is available in many different forms, which include data, text, image, voice, and signal. However, disparate information needs to be processed meticulously and intelligently for it to be useful. In this aspect, intelligent information processing systems play an important role to help extract meaningful and significant information from spurious one, and to help decision makers to make informed decisions.

Over the years, researchers have designed and developed a variety of intelligent information processing techniques, and have demonstrated their applicability to undertaking various complex problems. In this special issue, a total of seven articles in the general area of intelligent information processing are presented. It should be noted that these articles represent only a small part of the huge research activities in this exciting and fast growing area. The main aim is to highlight some recent advances in different intelligent information processing techniques and their applications, ranging from natural language processing, affective computing, data mining, to fuzzy information processing.

The first and second articles are concerned with natural language processing and affective computing. In the first article, an intelligent natural language processing technique that allows a machine to detect illogical word combinations is described. A knowledge model of human discourse and words, focusing on nouns and adjective phrases, is created. Using a tree-like structure, the knowledge model is used to form general properties of objects. An association model is then used to form word-to-word relationships based on a

*Corresponding author. E-mail: Lakhmi.jain@unisa.edu.au. database of approximately 120,000 words organized in sets of concepts and attributes. Applicability of the proposed technique to differentiating logical and illogical discourse texts in Japanese is demonstrated, and the answers from the machine and from humans are compared and analyzed. In the second article, an intelligent affective computing model that is able to judge the emotion of humans from uttered sentences is proposed. A large concept database, with approximately 90,000 concepts, and a mechanism to process the degree of association among the concepts are constructed. A knowledge base is then used to process sense and perception of judgment of human emotions. A total of 530 sentences have been collected in a case study to demonstrate the applicability of the emotion judgment system.

The third and fourth articles focus on intelligent data mining techniques and applications. An innovative technique to extract users' interests from bookbrowsing behaviors with RFID is presented in the third article. An extraction engine to predict users' interests using keywords in books and the browsing time of the books is developed. An experiment to show the correlation between the browsing time and users' interests in the books is conducted. The experimental results indicate that the proposed model is able to extract keywords that represent users' interests more precisely than the traditional method. In the fourth article, an intelligent algorithm to extract research communities from bibliographic data is described. A list of frequent keywords is obtained from the bibliographic data, and the algorithm is used to compute the relationship between research communities and the keywords. An experiment to demonstrate the applicability of the proposed algorithm to finding research communities from the CiteSeer bibliographic database, which contains over 
700,000 entries of research documents, is conducted. The results show that more compact and close communities are produced by the proposed algorithm, as compared with those from other methods reported in the literature.

The last three articles cover research works in fuzzy information processing. In the fifth paper, a fuzzy hyper-prototype clustering algorithm is explained. The main aim is to find a partition matrix and a group of hyperplanes that minimize the sum of weighted distances from all data samples to the cluster centers. Casting the clustering problem as a constrained optimization problem, a fuzzy objective function is formulated, and an iterative algorithm is used to update the cluster centers and the partition matrix until convergence is achieved. The performance of the proposed fuzzy algorithm is evaluated using simulated and benchmark data sets, and superior results as compared with those from existing methods are reported. In the sixth article, a fuzzy model with two distinct types of fuzzy rules for decision making in automated call management in a cellular mobile network is presented. A fuzzy comparator is deployed to compare the membership functions of two fuzzy measurement variables. Two approaches, based on fuzzy to binary mapping and fuzzy to fuzzy mapping, to handle the problem are explained. Application of the proposed fuzzy model to undertaking both channel assignment and call admission control as a combined problem is demonstrated. The results indicate that the proposed fuzzy model is able to better manage and serve incoming calls in a cellular network than the existing techniques.

A learning based fuzzy clustering method is described in the last article. Its key feature is that noise is included in the learning process of a traditional selforganized additive fuzzy clustering method. The similarity measure used consists of two parts, one is a self-organized similarity, and another is the similarity between the self-organized similarity and noise of the model. By learning the status of noise, the proposed method is able to obtain more adaptable results. Applicability and effectiveness of the proposed method to electroencephalogram (EEG) data analysis is demonstrated, and clearer classification structures when compared with those from other fuzzy clustering models are obtained.

The guest editors are grateful to (i) the authors for their contributions; (ii) the reviewers for their time and effort in reviewing the manuscripts; (iii) the journal production team for their support and help in producing this special issue. 